\title{
Persistent trigeminal artery as a rare cause of ischaemic lesion and migraine-like headache
}

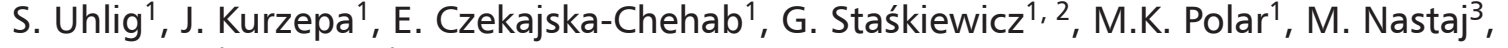 \\ E. Stochmal' ${ }^{1}$ A. Drop ${ }^{1}$ \\ ${ }^{1} 1^{\text {st }}$ Department of Radiology, Medical University of Lublin, Poland \\ ${ }^{2}$ Department of Human Anatomy, Medical University of Lublin, Poland \\ ${ }^{3}$ Department of Neurology, University Hospital No. 4 in Lublin, Poland
}

[Received 2 June 2014; Accepted 23 July 2014]

The persistent trigeminal artery (PTA) is a rare remnant of the embryonic intracranial circulatory system that forms a carotid-vertebrobasilar anastomosis. In most cases PTA does not have clear clinical implications. However, some authors report the association of PTA occurrence with vertigo, dizziness and nerve palsy, resulting in diplopia, strabismus or trigeminal neuralgia in patients. In rare cases it may also be related to posterior cerebral circulation strokes. This work reports the case of a female patient who presented with migraine-like headache and an ischaemic lesion in the left temporal lobe in association with PTA. (Folia Morphol 2015; 74, 1: 133-136)

Key words: persistent trigeminal artery, migraine-like headache, steal phenomenon, computed tomography angiography, computed tomography angiography

\section{INTRODUCTION}

Arterial foetal anastomoses exist between the carotid artery and vertebrobasilar system during the early stages of embryogenesis. These anastomoses are made by the trigeminal, otic, hypoglossal and proatlantal intersegmental arteries [1,3]. Before the vertebral and posterior communicating arteries develop, the trigeminal artery temporarily supplies the hindbrain at about 28-35 days of gestation. The trigeminal artery becomes unnecessary after the vertebrobasilar system forms and involutes together with the three remaining vessels [12]. These anastomoses occasionally persist in postnatal life, resulting in one of many possible malformations of the circle of Willis and the persistent trigeminal artery (PTA) is the largest and the most frequent among them $[9,16]$.

In patients undergoing vascular examinations, PTA and its variants are reported with a frequency of $0.1-1 \%$, depending on the imaging modality used $[3,17]$. In early angiographic studies, the frequency of PTA in examined groups was approximately $0.2 \%$ [25]; however, in newer reports this number is significantly higher. A study published by Allen et al. [2] in 2005 showed that out of 481 routine cerebral angiograms, PTA was detected in 5 (1\%) patients. In magnetic resonance (MR) angiography examinations, PTA with its variants is detected in about $0.5 \%$. Uchino et al. [24] reported that out of $3626 \mathrm{MR}$ angiographic examinations, $12(0.34 \%)$ cases of PTA and $6(0.17 \%)$ cases of PTA variants were found. Another large study examining 16,415 MR angiographies revealed that the incidence of both PTA and its variants was $0.68 \%$ [18].

The PTA usually arises from the cavernous segment of the presellar internal carotid artery, courses posteriorly in proximity to the Gasserian ganglion and joins the distal third of the basilar artery. This usually

Address for correspondence: Dr G. Staśkiewicz, $1^{\text {st }}$ Department of Radiology, ul. Jaczewskiego 8, 20-954 Lublin, Poland, tel: +48 81724 46 60, fax: +48 8172445 43, e-mail: grzegorz.staskiewicz@gmail.com 
occurs between the origins of the superior cerebellar arteries and anterior inferior cerebellar arteries; however, there are different types of this vessel.

Saltzman [21] proposed the angiographic classification of PTA according to the configuration of the ipsilateral posterior cerebral artery. The type I is characterised by the lack of the posterior communicating artery, which in normal conditions supplies the vertebrobasilar system distal to the anastomosis. In Saltzman type II, the ipsilateral posterior communicating artery origins from the internal carotid artery with aplasia of the P1 segment. In both Saltzman types, the part of the basilar artery distal to the PTA is usually hypoplastic [9]. Saltzman type III (IIIa, IIIb, IIIc) corresponds to a PTA variant when the anastomosis joins a cerebellar artery directly. Salas et al. [20] provided another classification system of PTA. The authors divided PTA into two types based on their relationships to the lateral and medial abducent nerve. Both types occur with nearly equal frequency [9]. In the lateral type, also known as the petrosal variation, the artery courses posterolaterally and runs together with the sensory roots of the trigeminal nerve. In the medial type, also known as the sphenoidal variation, the artery has an intrasellar or transhypophyseal course near the pituitary gland, penetrating the dorsum sella. Both classification systems are used today.

In the majority of cases, PTA does not have clinical implications; however, some publications reveal the coincidence of PTA with vertigo, ataxia and other neurological symptoms $[4,10]$. Its relation to migraine-like headaches is not well documented; however, in recent years, a few publications concerning anomalies of intracranial arteries or vascular malformations as a cause of migraine symptoms appeared $[7,8,19]$. In rare cases PTA can contribute to cerebral stroke, which may be induced with thrombotic changes or steal syndrome [1]. The presence of PTA may also be associated with a higher likelihood of concomitant vascular pathologies, such as: arteriovenous malformations, carotid cavernous fistulas, moyamoya disease or abnormalities of the aortic arch and its branches $[3,9,17]$.

This report presents a case of persistent trigeminal artery in a female patient as a possible cause of migraine-like headache and ischaemic lesion in temporal lobe.

\section{CASE REPORT}

A 41 -year-old female was admitted to the Neurology Department at our hospital because of re- current headaches. The attacks have begun many years ago, occurring 3-5 times per month and persisted for 2-3 days. The headaches were mostly left-sided with symptoms occasionally presenting on the opposite side. Almost each episode was accompanied with photophobia, phonophobia, nausea, and vomiting. Additionally, there were associated facial paresthesias ipsilateral to the headache. The episodes were triggered and worsened by physical exertion. Neurological examination was normal and the patient denied additional symptoms. To exclude cerebral pathologies the decision to perform computed tomography angiography (CTA) was made.

The examination was performed on a 64-row CT scanner (LightSpeed VCT GE Healthcare). Raw data was reconstructed and assessed using Advantage Window 4.6 dedicated console. During native scanning, the region of the skull base was scanned with collimation of $2.5 \mathrm{~mm}(140 \mathrm{kV}, 335 \mathrm{~mA})$ and remaining structures of the brain were scanned with collimation of $5 \mathrm{~mm}$ ( $120 \mathrm{kV}, 320 \mathrm{~mA})$. CTA examination of the brain was performed with $80 \mathrm{~mL}$ of intravenously administered non-ionic contrast $(370 \mathrm{mg} / \mathrm{mL})$, infused at a rate of $4 \mathrm{~mL} / \mathrm{s}$. Delay time was determined with Smart Prep technique and scanning parameters included collimation of $0.625 \mathrm{~mm}, 120 \mathrm{kV}$, and $330 \mathrm{~mA}$, tube rotation time $0.5 \mathrm{~s}$.

Native scanning revealed an ischaemic lesion in the peri-insular region of the left temporal lobe (Fig. 1). In CTA series a left-sided persistent trigeminal artery was found (Fig. 2). The vessel originated from the $\mathrm{C} 4$ section of the internal carotid artery where its diameter was $3.4 \mathrm{~mm}$. It took a posterolateral course, encircling the dorsum sella. Distal to this, it coursed towards the petrous part of the temporal bone, where it penetrated into its apex. It continued medially merging eventually with the upper part of the basal artery just below superior cerebellar arteries. The diameter at the distal end of the PTA was $2.1 \mathrm{~mm}$ and its total length was $27 \mathrm{~mm}$. The caudal portion of basal artery was hypoplastic. Morphology and course of the PTA is an example of a Saltzman type I and according to the Salas classification it corresponded to the lateral variation.

Additionally, the right $A 1$ segment was hypoplastic while the right posterior communicating artery and anterior communicating artery were well developed. The left posterior communicating artery was not visible in CTA examination. 

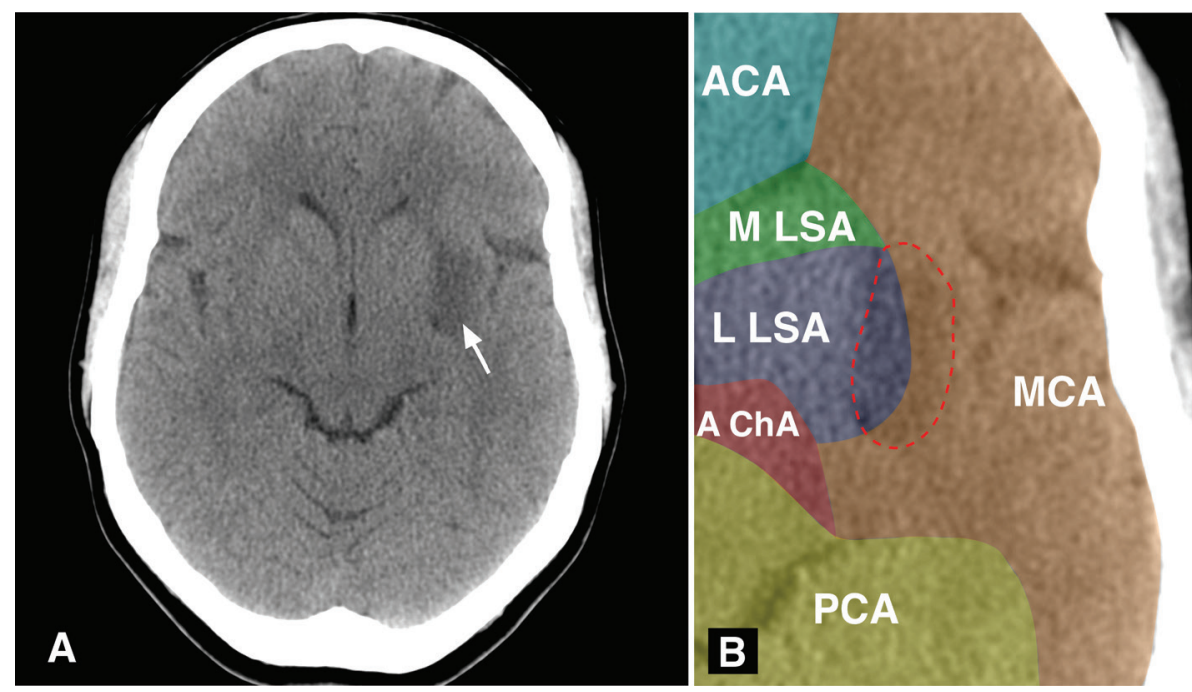

Figure 1. A. Axial scan presenting ischaemic lesion in left temporal lobe indicated with arrow; B. Area in the neighbourhood of ischaemic lesion (bordered with red dashed line) is enlarged and vascular territories are marked with colours: orange - medial cerebral artery (MCA), cyan — anterior cerebral artery (ACA), yellow — posterior cerebral artery (PCA), green - medial lenticulostriate arteries (M LSA) (ACA branches), navy blue — lateral lenticulostriate arteries (L LSA) (MCA branches), red — anterior choroidal arteries (AChA).
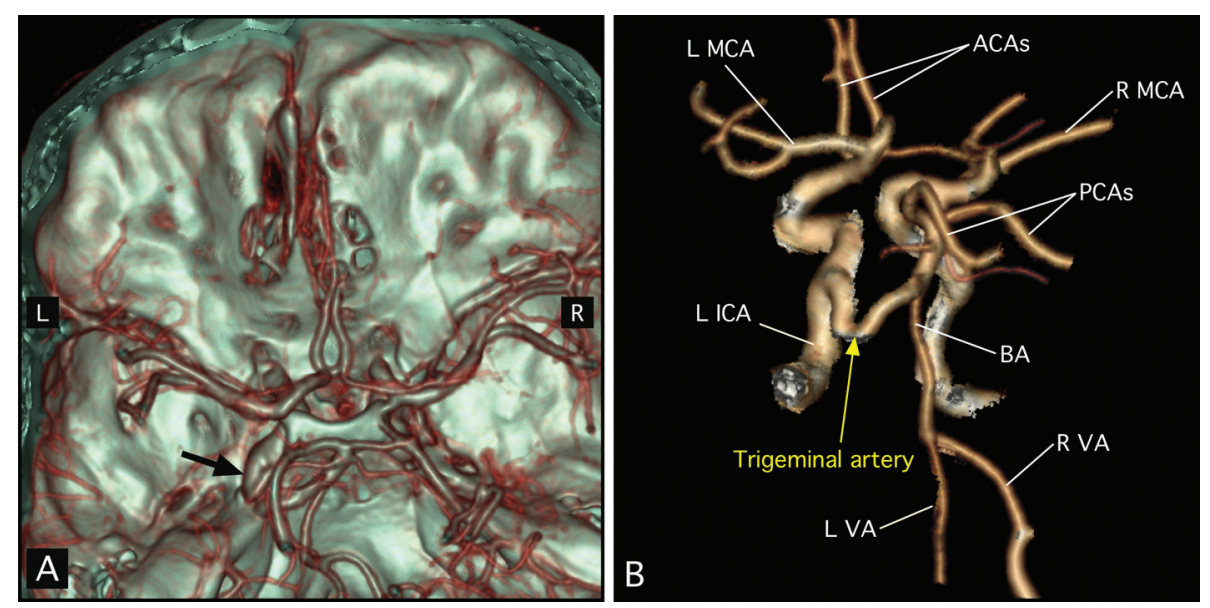

Figure 2. A. Computed tomography angiography with visible persistent trigeminal artery indicated with arrow; B. Circle of Willis in volume rendering with bone extraction option; VA — vertebral arteries (left and right); BA — basal artery; LICA — left internal carotid artery; PCAs — posterior cerebral arteries; ACAs — anterior cerebral arteries; MCA — middle cerebral arteries (left and right). Trigeminal artery indicated with yellow arrow.

Based on CTA examination and clinical symptoms, migraine-like headache due to PTA was diagnosed and the decision to treat conservatively was made. Currently, the patient is treated with iprazochrome $(2.5 \mathrm{mg}) 3$ times per day. During treatment the headaches appear only 1-2 times per month and are less intense.

\section{DISCUSSION}

In the majority of cases, PTA is an incidental finding on brain imaging, however, it was also previously described as a rare cause of various neurological symptoms such as $6^{\text {th }}$ or oculomotor nerve palsies [4], vertigo, dizziness [10], ataxia and stroke [13]. The significance of PTA in occlusive cerebrovascular disease, especially brainstem transient ischaemic attack or infarcts and occipital infarcts is discussed. The pathogenesis of vertebrobasilar insufficiency in patients with PTA may be attributed to embolism from an atherosclerotic internal carotid artery via PTA. There may also be rare cases of PTA thrombosis leading to decreased blood flow in the basilar artery $[11,22]$. Carotid artery insufficiency secondary to stenosis, dissection or transitional haemodynamic 
changes can lead to a vascular steal phenomenon from the basilar artery to the carotid system through a persistent trigeminal artery $[1,3]$. In the presented case, morphology of the ischaemic lesion suggested it was caused by chronic haemodynamic insufficiency. However, its localisation was not typical, occurring in the region corresponding to the border zone between the area vascularised by peripheral branches of the middle cerebral artery and lateral lenticulostriate arteries (Fig. 1) [15]. Therefore, it could suggest the existence of steal syndrome with reverse direction of blood flow, from carotid artery to vertebrobasilar system, being a possible cause of watershed ischaemia. With the exception of facial paraesthesia occurring during episodes of headache, the patient had no other clinical symptoms related to the detected lesion.

PTA can often lead to recurrent neuralgic-type head pain. The proximity of Gasser's ganglion and the trigeminal nerve may lead to neurovascular conflict resulting in trigeminal neuralgia [6]. Based on published data, PTA is observed approximately 4 times more often in patients with trigeminal neuralgia [1].

There is, however, little evidence linking the presence of PTA and migraine-like headache. According to The International Classification of Headache Disorders $2^{\text {nd }}$ Edition, migraine-like headaches can be provoked by other disorders, but cases of such episodes related to carotid-vertebrobasilar anastomoses are not numerous [23]. In a pictorial essay describing the embryological aspects of such anastomoses, Luh et al. [14] cites 2 cases in which the patients underwent MR imaging examination for headache. In the first case, PTA was found, while in the second case, a persistent hypoglossal artery was detected. In 2011, Clerici et al. [5] described the case of a 56-year-old male with an episode of acute headache related to a persistent hypoglossal artery, which was a likely cause of temporal haemodynamic vulnerability of the posterior circulation. The mechanism of headaches in the patient we describe could be similar; however, there were no apparent symptoms related to insufficiency of the posterior circulation such as vertigo or vision disturbances. This supports the theory of a steal phenomenon from the carotid artery to the vertebrobasilar system.

\section{CONCLUSIONS}

In conclusion, PTA, among many other anomalies of intracranial vessels, should be considered as a potential cause of migraine-like headaches. It can also be the cause of temporal haemodynamic vulnerabilities leading to chronic ischaemic lesions.

\section{REFERENCES}

1. Alcalá-Cerra G, Tubbs RS, Niño-Hernández LM (2012) Anatomical features and clinical relevance of a persistent trigeminal artery. Surg Neurol Int, 3: 111.

2. Allen JW, Alastra AJ, Nelson PK (2005) Proximal intracranial internal carotid artery branches: Prevalence and importance for balloon occlusion test. J Neurosurg, 102: 45-52.

3. Azab W, Delashaw J, Mohammed M (2012) Persistent primitive trigeminal artery: a review. Turk Neurosurg, 22: 399-406.

4. Bosco D, Consoli D, Lanza PL, Plastino M, Nicoletti F, Ceccotti C (2010) Complete oculomotor palsy caused by persistent trigeminal artery. Neurol Sci, 31: 657-659.

5. Clerici AM, Craparo G, Cafasso G, Micieli C, Bono G (2011) De-novo headache with transient vertebro-basilar symptoms: role of embryonic hypoglossal artery. J Headache Pain, 6: 639-643.

6. Clerici AM, Merlo P, Rognone F, Noce M, Rognone E, Bono G (2009) Persistent trigeminal artery causing "double" neurovascular conflict. Headache: J Head Face Pain, 3: 472-476.

7. Cucchiara B, Detre J (2008) Migraine and circle of Willis anomalies. Med Hypotheses, 70: 860-865.

8. Cucchiara B, Wolf RL, Nagae L, Zhang Q, Kasner S, Datta R, Aguirre GK, Detre JA (2013) Migraine with aura is associated with an incomplete circle of willis: Results of a prospective observational study. PloS One, 8: e71007.

9. Dimmick SJ, Faulder KC (2009) Normal variants of the cerebral circulation at multidetector CT angiography. Radiographics, 29: 1027-1043.

10. Eluvathingal Muttikkal TJ, Varghese SP, Chavan VN (2007) Persistent trigeminal artery and associated vascular variations. Australas Radiol, 51 Spec No.: B31-B33.

11. Iancu $D$, Anxionnat $R$, Bracard S (2010) Brainstem infarction in a patient with internal carotid dissection and persistent trigeminal artery: a case report. BMC medical imaging, 10: 14

12. Kathuria S, Gregg L, Chen J, Gandhi D (2011) Normal cerebral arterial development and variations. Semin Ultrasound CT MR, 32: 242-251.

13. Kwon JY, Lee EJ, Kim JS (2010) Brainstem infarction secondary to persistent trigeminal artery occlusion: Successful treatment with intravenous rt-PA. Eur Neurol, 64: 311.

14. Luh GY, Dean BL, Tomsick TA, Wallace RC (1999) The persistent fetal carotid-vertebrobasilar anastomoses. Am J Roentgenol, 5: 1427-1432.

15. Maas MB, Safdiech JE (2009) Ischemic stroke: Pathophysiology and principles of localization. Neurology, 13 (Part 1): 2-16.

16. Makowicz G, Poniatowska R, Lusawa M (2013) Variants of cerebral arteries: anterior circulation. Pol J Radiol, 78: 42-47.

17. Meckel S, Spittau B, Mcauliffe W (2013) The persistent trigeminal artery: development, imaging anatomy, variants, and associated vascular pathologies. Neuroradiology, 55: 5-16.

18. O'uchi E, O'uchi T (2010) Persistent primitive trigeminal arteries (PTA) and its variant (PTAV): Analysis of 103 cases detected in 16,415 cases of MRA over 3 years. Neuroradiology, 52: 1111-1119.

19. Obermann M, Gizewski ER, Limmroth V, Diener HC, Katsarava Z (2006) Symptomatic migraine and pontine vascular malformation: Evidence for a key role of the brainstem in the pathophysiology of chronic migraine. Cephalalgia, 26: 763-766.

20. Salas E, Ziyal IM, Sekhar LN, Wright DC (1998) Persistent trigeminal artery: An anatomic study. Neurosurgery, 43: 557-561.

21. Saltzman GF (1959) Patent primitive trigeminal artery studied by cerebral angiography. Acta Radiol, 51: 329-336.

22. Schwartz NE, Albers GW (2007) Neurological picture. Acute strokes in the setting of a persistent primitive trigeminal artery. J Neurol Neurosurg Psychiatry, 78: 745.

23. Subcommittee IHSC (2004) International classification of headache disorders, 2nd edition. Cephalalgia, 24 (suppl 1): 1-160.

24. Uchino A, Saito N, Okada Y, Kozawa E, Mizukoshi W, Inoue K, Takahashi M (2012) Persistent trigeminal artery and its variants on MR angiography. Surgical and radiologic anatomy, 34: 271-276.

25. Wise BL, Palubinskas AJ (1964) Persistent trigeminal artery (carotid-basilar anastomosis). J Neurosurg, 21: 199-206. 